\title{
Measurement and simulation of surface roughness noise using phased microphone arrays *
}

\author{
Y. Liu* ${ }^{*}$, A.P. Dowling, H.-C. Shin \\ Department of Engineering, University of Cambridge, Trumpington Street, \\ Cambridge CB2 1PZ, UK
}

\begin{abstract}
A turbulent boundary-layer flow over a rough wall generates a dipole sound field as the near-field hydrodynamic disturbances in the turbulent boundary-layer scatter into radiated sound at small surface irregularities. In this paper, phased microphone arrays are applied to the measurement and simulation of surface roughness noise. The radiated sound from two rough plates and one smooth plate in an open jet is measured at three streamwise locations, and the beamforming source maps demonstrate the dipole directivity. Higher source strengths can be observed on the rough plates which also enhance the trailing-edge noise. A prediction scheme in previous theoretical work is used to describe the strength of a distribution of incoherent dipoles and to simulate the sound detected by the microphone array. Source maps of measurement and simulation exhibit satisfactory similarities in both source pattern and source strength, which confirms the dipole nature and the predicted magnitude of roughness noise. However, the simulations underestimate the streamwise gradient of the source strengths and overestimate the source strengths at the highest frequency.
\end{abstract}

Key words: surface roughness noise, aeroacoustic dipole, phased microphone array, beamforming algorithm.

\footnotetext{
* Submitted to Journal of Sound and Vibration on 14 May 2007, accepted for publication on 21 December 2007. Original version presented in the 13th AIAA/CEAS Aeroacoustics Conference, Rome, Italy, 21-23 May 2007.

* Corresponding author. Tel.: +44 1223 766063; fax: +44 1223330282.

Email address: y1275@cam.ac.uk (Y. Liu).
} 


\section{Nomenclature}

a monopole source strength

$A$ area of the rough region

$c \quad$ speed of sound in free field

$d \quad$ distance between microphones and their nearest neighbour

$D \quad$ directivity function

$f \quad$ frequency

i $\quad \sqrt{-1}$

$I_{1}, I_{2}$ integrals with respect to the wavenumber vector $\boldsymbol{\kappa}$

$k \quad$ acoustic wavenumber

$l \quad$ distance between the two coherent monopoles of a dipole

$M \quad$ free stream Mach number

$n \quad$ number of frequency intervals

$N \quad$ average number of roughness bosses per unit area

$\hat{p} \quad$ acoustic frequency spectrum

$P \quad$ power spectral density of $\hat{p}$

$P_{R} \quad$ power spectral density of far-field radiated roughness noise spectrum

$P_{s} \quad$ smooth-wall wavenumber-frequency spectrum

$r \quad$ propagation distance

$R \quad$ roughness height

$R e_{\tau} \quad$ roughness Reynolds number, $R e_{\tau}=R u_{\tau} / \nu$

$S h^{*} \quad$ Strouhal number, $S h^{*}=\omega \delta^{*} / U$

$u_{\tau} \quad$ friction velocity

$U$ free stream velocity

$U_{c} \quad$ eddy convection velocity

$x_{1} \quad$ streamwise distance from the front edge of rough region

$x, y, z$ Cartesian coordinates

$\delta \quad$ boundary-layer thickness

$\delta^{*} \quad$ displacement boundary-layer thickness

$\delta($.$) \quad Dirac delta function$

$\boldsymbol{\kappa} \quad$ wavenumber vector, $\boldsymbol{\kappa}=\left(\kappa_{1}, 0, \kappa_{3}\right)$

$\Lambda \quad$ power spectral density of $a$

$\mu \quad$ roughness density factor, $\mu=1 /\left(1+\frac{1}{4} \sigma\right)$

$\nu \quad$ kinematic viscosity

$\omega \quad$ radian frequency

$\Phi \quad$ point pressure frequency spectrum

$\rho_{0} \quad$ mean fluid density in free field

$\sigma \quad$ roughness density

$\tau_{w} \quad$ mean wall shear stress, $\tau_{w}=\rho_{0} u_{\tau}^{2}$

$\theta, \phi \quad$ directivity angles

- mean value

- ensemble average

,+- monopoles with opposite phase

1,2 dipoles $\mathrm{DPL}_{1}$ and $\mathrm{DPL}_{2}$ 
$i, j \quad$ general summation variable

tot total power

$\mathrm{DPL}_{1}$ dipole in flow direction

$\mathrm{DPL}_{2}$ dipole normal to flow direction

CFT continuous Fourier transform

DFT discrete Fourier transform

FFT fast Fourier transform

SPL sound pressure level

\section{Introduction}

The generation of sound by turbulent boundary-layer flow at low Mach number over a rigid rough wall has been investigated analytically by Howe [1-4]. In Howe's diffraction theory, the rough surface was modelled by a random distribution of rigid, hemispherical bosses over a rigid plane. The near-field hydrodynamic disturbances in the turbulent boundary layer scatter into radiated sound due to the presence of small surface irregularities. The roughness elements behave like point dipoles with the dipole strength due to scattering of the near turbulent pressure fluctuations on an element, and thus surface roughness noise is likely to be dominant at low Mach number. Howe [1] also speculated that roughness noise would be a substantial fraction of the airframe noise of an airplane flying in the "clean" configuration in which the landing gears and high-lift devices are stowed.

In the theoretical model [1], the acoustic frequency spectrum $P_{R}(\omega)$ of far-field radiated roughness noise was expressed as an infinite integral in terms of the smooth-wall wavenumber-frequency spectrum $P_{s}(\boldsymbol{\kappa}, \omega)$. Howe evaluated $P_{R}(\omega)$ by means of a conventional asymptotic approximation [1,3] based on $P_{s}(\boldsymbol{\kappa}, \omega)$ being sharply peaked in the vicinity of the convective ridge. He also proposed empirical models [3-5] for $P_{R}(\omega)$ by curve-fitting available experimental data of Hersh [6]. The empirical coefficients were partially estimated, but not all the coefficient values could be determined due to the lack of directivity information in Hersh's data. Therefore, Howe's empirical models are unable to predict the absolute level of the rough-wall acoustic frequency spectrum $P_{R}(\omega)$.

The theoretical model of Howe [1] was recently extended by Liu and Dowling [7] to quantify numerically the far-field radiated roughness noise. They approximated the rough-wall wavenumber-frequency spectrum by smooth-wall models [8-12] with increased friction velocity $u_{\tau}$ and boundary-layer thickness $\delta$ appropriate for a rough surface. The infinite integral in the determination of $P_{R}(\omega)$ was evaluated by direct numerical integration. Their prediction scheme is able to reproduce the spectral characteristics of Howe's empirical models and Hersh's experimental data, and to predict appropriately the absolute level of 
far-field radiated roughness noise.

Liu and Dowling [7] then applied the numerical method to the assessment of the contribution of surface roughness to airframe noise, and estimated the roughness noise for a Boeing-757 sized aircraft wing during approach or take-off with idealized levels of surface roughness. They found that in the high frequency region sound radiated from surface roughness may exceed that from the trailing edge depending on the size of roughness elements, and that the trailing-edge noise is also enhanced by surface roughness to some extent. Therefore they suggested that the contribution of surface roughness to airframe noise needs to be considered in the design of a low-noise airframe.

However, few experiments have been performed so far to investigate the noise radiated from rough surfaces. Measurement of roughness noise is difficult as it can be easily contaminated due to its very low spectral levels. Hersh [6] studied the radiation of sound from sand-roughened pipes of various grit sizes. He concluded through measurements that the 6th power variation of the overall sound pressure level with flow velocity suggests a dipole source. However, the data are insufficient for building an empirical model, as aforementioned, due to the unknown effects of acoustic refraction by the free-jet shear layers downstream of the nozzle exit. Liu and Dowling [7] measured the noise spectra of two rough plates with different roughness heights in an open jet. The measured noise spectra were significantly contaminated by background noise, but the roughness noise was detected in $1-2.5 \mathrm{kHz}$ frequency range. The reasonable amount of agreement between measurement and prediction provided a preliminary validation of their numerical prediction scheme which was based on Howe's theoretical model [1].

In this paper, phased microphone arrays were applied to the experimental study of surface roughness noise. The radiated sound from two rough plates and one smooth plate in an open jet was measured by both high- and lowfrequency arrays. The source locations and source strengths of possible dipoles due to roughness elements on a rigid plate were identified and discussed. Acoustic measurements were performed at three streamwise locations to explore the directivity features of possible dipole sources. Theoretical predictions for the source distributions on the two rough plates were also obtained using Liu and Dowling's model [7].

However, the array measurements can be misinterpreted if applied directly to determine the locations and relative strengths of these distributed dipoles, and this is due to the assumption of distributed incoherent monopoles in the standard beamforming algorithm [13-15]. The technique developed by Jordan et al. [16] to account for the propagation characteristics of a single dipole is not applicable because the roughness dipoles are distributed over such a large region that the directivity varies considerably. The array analysis source code 
is inaccessible and so it was not possible to modify the algorithm to base the beamforming on dipole sources.

Instead, we processed the theoretical cross-power spectral densities through the same beamforming algorithm as the experimental data and compared the resulting predicted and measured source maps. This is equivalent to comparing theory and experiment after applying a filter which suppresses much the extraneous noise in the experiments. A distribution of incoherent dipoles was simulated over a rigid plate with the source strengths determined equivalently by the prediction scheme of Liu and Dowling [7]. Comparison of the results of beamforming between measurement and simulation provides indirect validation of the predicted source type, magnitude and distribution.

\section{Experimental setup}

\subsection{Test rough plate}

The experiments were conducted in the open jet of a low-speed wind tunnel in the Whittle Laboratory of the Department of Engineering, Cambridge University, as illustrated in Fig. 1. The wind tunnel has an inner cross section of $0.586 \times 0.350 \mathrm{~m}^{2}$ at the outlet and a velocity range of $0-31.0 \mathrm{~m} \mathrm{~s}^{-1}$. Plastic foam lined in the inner walls and a splitter silencer was installed to reduce the wind noise and motor noise traveling inside the tunnel. A large flat plate made of aluminium alloy is placed nominally in the vertical meridian plane of the open jet. It is secured to a vertical frame of aluminium rods adjacent to the tunnel outlet. Fig. 1(b) shows that the plate surface is partially roughened in a rectangular region by a square distribution of hemispherical bosses. This was achieved by machining a recess of $0.64 \times 0.64 \mathrm{~m}^{2}$ into the plate surface. Four modeling panels of $0.32 \times 0.32 \mathrm{~m}^{2}$ with rigid, hemispherical plastic beads in parallel columns were flush mounted in the recess to form a rough region. Four smooth panels were also fabricated for the measurement of a smooth plate or a rough plate with a smaller rough region.

Three different surface conditions were examined:

(1) Rough1, $R=4 \mathrm{~mm}, \sigma=0.50$;

(2) Rough2, $R=3 \mathrm{~mm}, \sigma=0.44$;

(3) Smooth, $R=0 \mathrm{~mm}, \sigma=0.0$;

where $R$ and $\sigma$ are the roughness height and roughness density [1,7], respectively. 


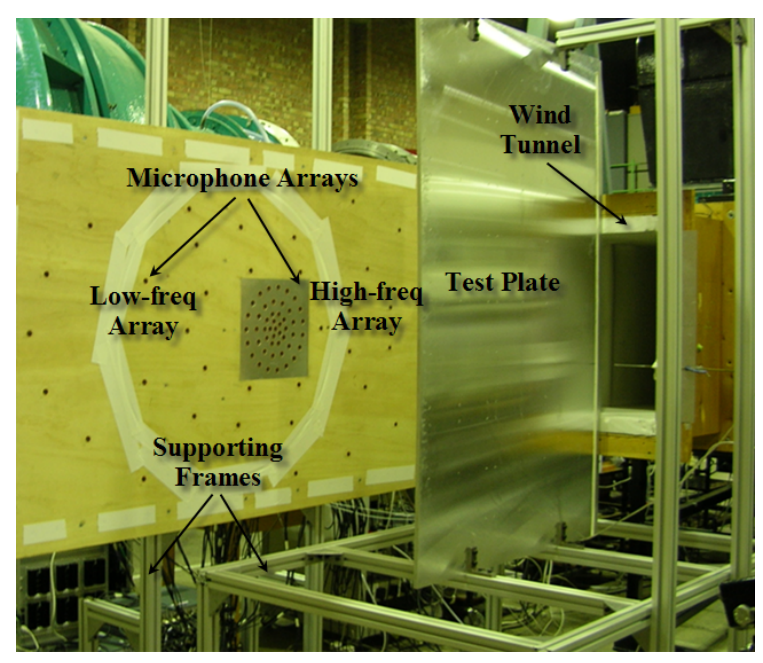

(a)

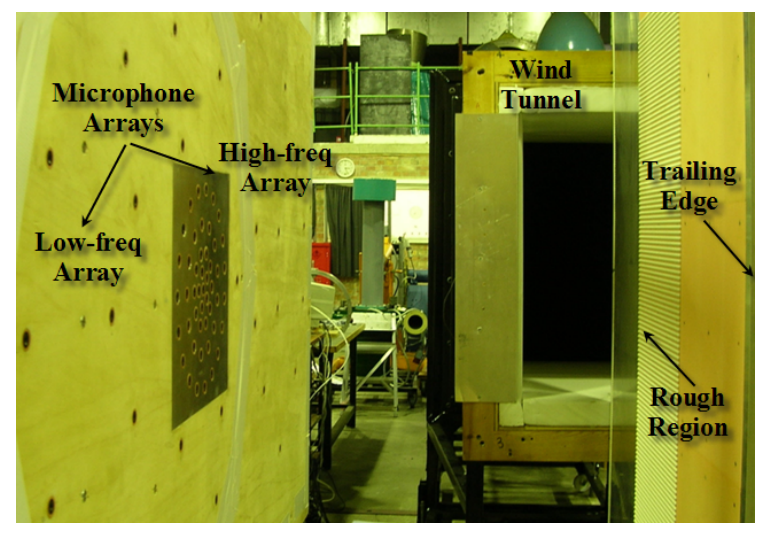

(b)

Fig. 1. Schematic of the experimental setup: (a) overview; (b) closeup.

The rough region is located at $0.34 \mathrm{~m}$ from the leading edge of the test plate, where the turbulent boundary layer is tripped, to ensure that the roughness elements are contained entirely within the boundary layer $[1,7]$ and to avoid the interference of sound scattering at the leading edge. Acoustic measurements were performed at free stream flow velocities, $U=15,20,25$ and $30 \mathrm{~m} \mathrm{~s}^{-1}$. The roughness noise scales as $U^{6}$ and so is more detectable at the higher velocity [7]. Therefore the experimental results discussed in $\S 3$ are for the velocity $U=30 \mathrm{~m} \mathrm{~s}^{-1}$ when differences between the acoustic data of the rough and smooth plates are most evident. As a precaution, all measurements were made when the laboratory ventilation system was not in operation.

\subsection{Phased microphone array}

Phased microphone arrays were utilized to localize the possible dipole sources in the rough region. The advantage of phased microphone arrays lies in the 
Table 1

Overall array dimensions and the maximum and minimum values of $d$ for each array.

\begin{tabular}{ccccc}
\hline Microphone array & Length $(\mathrm{m})$ & Width $(\mathrm{m})$ & $d_{\min }(\mathrm{m})$ & $d_{\max }(\mathrm{m})$ \\
\hline High-frequency & 0.25 & 0.25 & 0.023 & 0.044 \\
Low-frequency & 1.77 & 0.89 & 0.081 & 0.238 \\
\hline
\end{tabular}

improved signal-to-noise ratio, and hence noise sources below the background noise can be identified. In the present study, both the high- and low-frequency arrays were used each of which consists of 48 microphones located on optimized concentric circles or ellipses and flush mounted in a rigid board. The microphones are positioned irregularly with nonuniform spacing. The microphones are packed more closely near the centre of the array, and are wider spaced towards the boundary. For each microphone, $d$ is defined as the distance to its nearest neighbour. Table 1 gives the overall array dimensions and the maximum and minimum values of $d$ for each array. As shown in Fig. 1(a), the array board is supported by an aluminium frame with wheels. The board plane is aligned parallel to the test plate and the distance between them is adjustable. The microphone arrays were located in the far field of each roughness element, but not in the far field of the entire rough region.

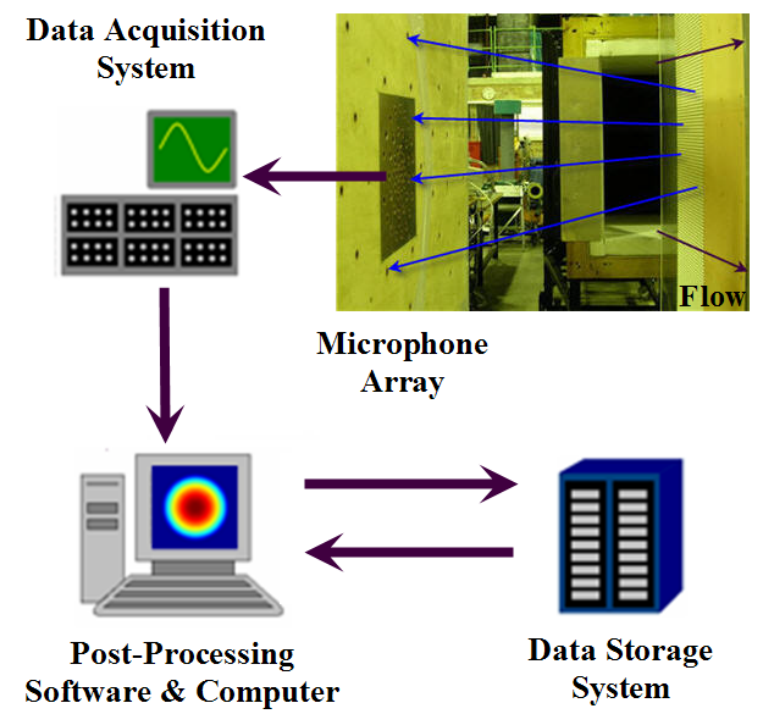

Fig. 2. Schematic of the acoustic measurement by a phased microphone array.

Figure 2 illustrates the schematic of the acoustic measurement by a phased microphone array. During a measurement the acoustic pressures were synchronously obtained by array microphones at a sampling frequency of $120 \mathrm{kHz}$ (high-frequency array) or $30 \mathrm{kHz}$ (low-frequency array) and a duration time of $60 \mathrm{~s}$. The raw data were transferred to the computer through the 48-channel data acquisition system and post-processed by the analysis software. Then source maps with sound pressure level (SPL) data were generated within 1/3 
octave-band frequencies by beamforming ${ }^{1}$ the post-processed data. The final data were sent to the 1.2 TB data storage system for future reference.

In the beamformer, the measured signals in the time domain are transformed to complex pressures in the frequency domain by the fast Fourier transform (FFT). The matrix of cross-power spectral densities between all microphone combinations is formulated in the frequency domain. The open-jet tunnel has some background noise and so we remove the diagonal elements of the matrix (i.e. the auto-power) and determine the monopole source strength at each element of the source grid that gives a best least-square fit to the measured cross-powers. First, a scanning grid containing the test plate is defined. The monopole source strength at each grid point is estimated by finding the value which gives the best match between measured cross-powers and the field of a monopole located at that grid point. The beamformer used the true distance from each source element to each microphone.

Obviously, the advantage of an open jet is to eliminate the reverberation noise of a closed-return wind tunnel. However, the uniform flow assumption of analysis software is not valid in the case of out-of-flow measurements in the test section of an open jet [17]. In this case, the effect of shear-layer refraction has to be incorporated in the source description of beamforming analysis. In fact for the low-speed wind tunnel in use $(M<0.1)$, although the propagating acoustic wave is somewhat refracted during transmission through the shear layer, the amplitude of received acoustic pressure by array microphones is almost unaltered, and hence the effect of shear-layer refraction on the predicted source strengths is negligible [15]. However, even a minor error in the signal phase will be amplified into a considerable distortion of source locations. In this study, the Amiet correction [18] for an infinitely thin shear layer was applied in beamforming analysis for the shear-layer correction.

\section{Results and discussion}

\subsection{Comparison of noise spectra}

Firstly, the comparison of the noise spectra for the rough and smooth plates is shown in Fig. 3. These noise spectra were measured by a pair of microphones at $0.3 \mathrm{~m}$ apart $(\bar{r}=1.2 \mathrm{~m}, \theta=\pi / 4, \phi=0$, see Fig. 8$)$. The cross-spectra data

\footnotetext{
1 "Beamforming" refers to an algorithm for the phased microphone array which, for each position on the source scanning plane, determines the monopole source strength at that location that best matches the data [13-15]. The beamforming code is implemented in the frequency domain.
} 


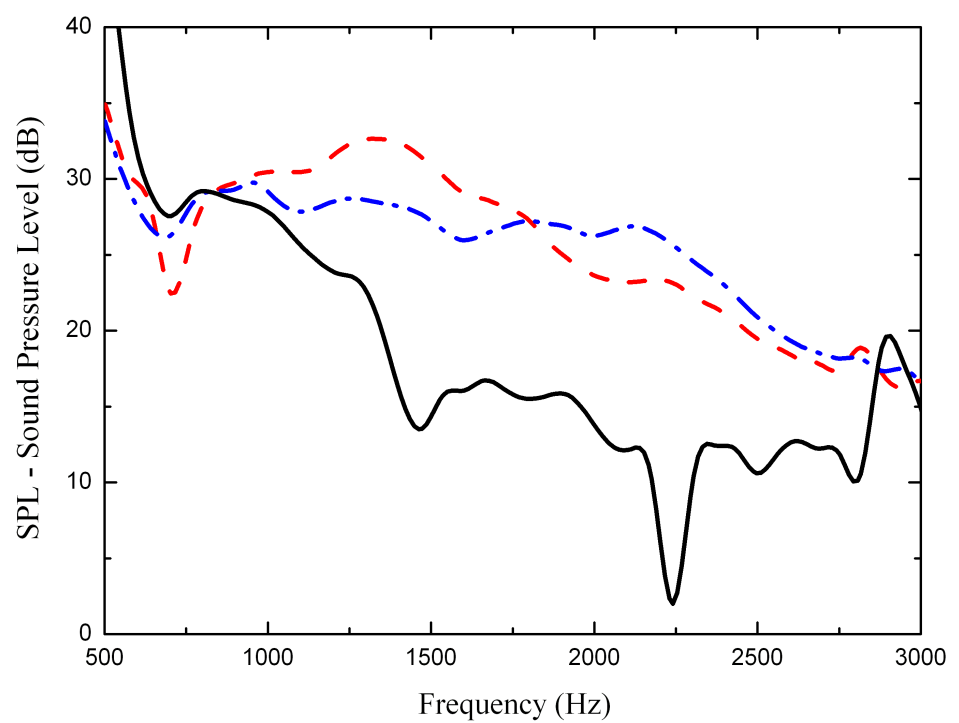

Fig. 3. Comparison of measured noise spectra: --- , Rough1; - - - Rough2; Smooth. $\Delta f=64 \mathrm{~Hz}$.

were post-processed in narrow band and divided by the bandwidth $\Delta f=64 \mathrm{~Hz}$ to give the spectral level.

As can be observed in Fig. 3, the experimental data are contaminated by background noise at low and high frequencies. The high SPL at the low frequencies $f<700 \mathrm{~Hz}$ is dominated by the wind noise propagating outwards from the tunnel, and the spectral peak around $f=2900 \mathrm{~Hz}$ is from the driving motor of the wind tunnel. The noise spectra of the rough plates are detected above the background noise, and are evidently higher than that of the smooth plate in the frequency range of $850-2850 \mathrm{~Hz}$. The spectral peak of Rough1 is above that of Rough2, but Rough2 produces more noise than Rough1 when $f>1800 \mathrm{~Hz}$ because the sound radiation from smaller roughness elements will be dominant at higher frequencies [7].

\subsection{Comparison of beamforming source maps}

The comparison of noise spectra in Fig. 3 suggests that it would be beneficial to focus the microphone array measurements within 1000-2500 Hz frequency range. Figs. 4 and 5 illustrate the beamforming source maps for the rough and smooth plates obtained by both high- and low-frequency arrays at location 2 (see Table 2). Three 1/3 octave-band frequencies are selected with centre frequencies $1250 \mathrm{~Hz}, 1600 \mathrm{~Hz}$ and $2000 \mathrm{~Hz}$, respectively.

In the beamforming analysis, the origin of the $x-y$ coordinates in all source maps is fixed at the array centre. The flow direction is from the left to the right. The inner dashed frame outlines the boundary of the rough region and the 

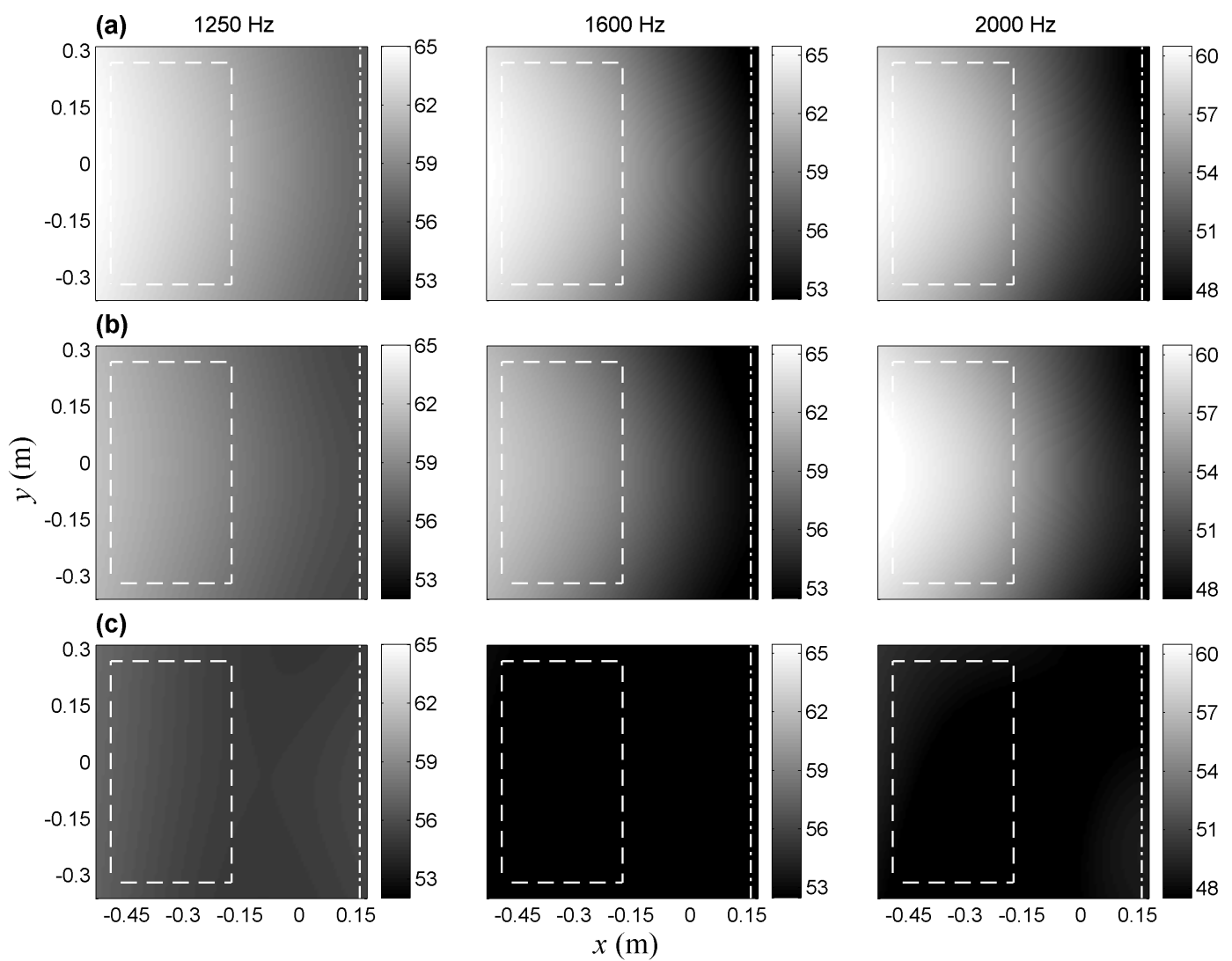

Fig. 4. Comparison of beamforming source maps: (a) Rough1; (b) Rough2; (c) Smooth. High-frequency microphone array.

dashdotted line downstream denotes the trailing edge. Note that in the current setup, only two rough panels were mounted into the $0.64 \times 0.64 \mathrm{~m}^{2}$ recess to form a smaller rough region upstream, which helps reduce the interference of sound scattering from the trailing edge. In addition, the source powers have been converted to SPL data at a reference distance of $1 / \sqrt{4 \pi} \mathrm{m}$ from the source [17]. The grey-scale bar gives the SPL in $\mathrm{dB}$, and the grey-scale bars for the rough and smooth plates at the same frequency are shown on identical scales for easy comparison. The dynamic ranges of the source maps obtained by the high- and low-frequency arrays are about $12 \mathrm{~dB}$ and $16 \mathrm{~dB}$, respectively.

As shown in Figs. 4 and 5, the source strengths on the rough plates exceed those on the smooth plate by about 10-15 dB. The source patterns of the two rough plates appear very similar with higher SPL for Rough1 at frequencies of 1250 and $1600 \mathrm{~Hz}$. However at $f=2000 \mathrm{~Hz}$, the source strengths of Rough2 exceed those of Rough1, which is consistent with the noise spectra data in Fig. 3. The major lobe of maximum source strengths occurs in the upstream portion of the rough region. This is because the ratio of roughness height to boundary-layer thickness, $R / \delta$, decreases as the boundary layer grows along the plate chord, which makes the downstream roughness elements less significant as sound scatterers. 
(a)

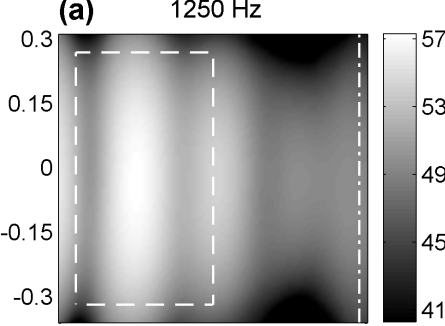

(b)

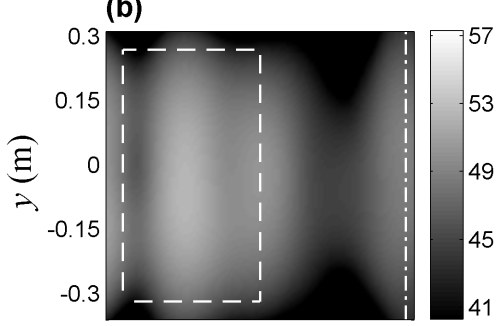

(c)

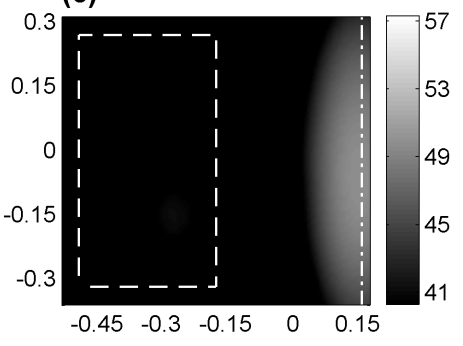

$1600 \mathrm{~Hz}$
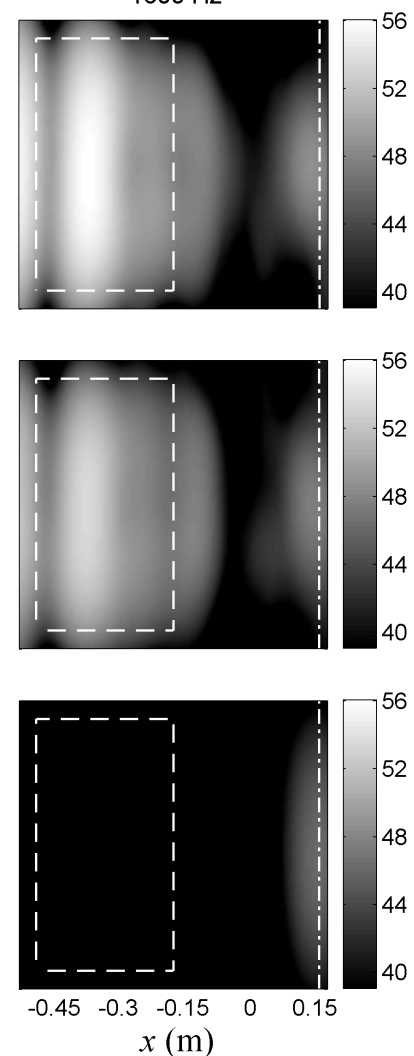

$2000 \mathrm{~Hz}$
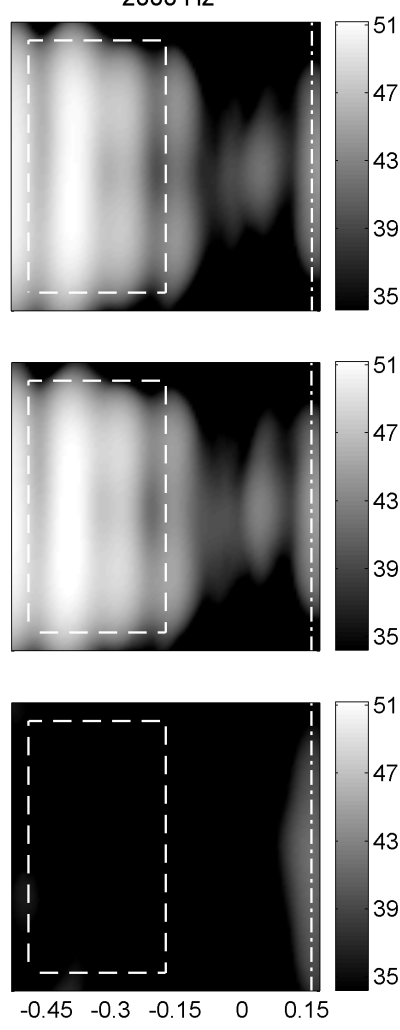

Fig. 5. Comparison of beamforming source maps: (a) Rough1; (b) Rough2; (c) Smooth. Low-frequency microphone array.

Comparing the source maps in Figs. 4 and 5, we find that the low-frequency array gives better resolution than the high-frequency array due to the selected low frequencies and the widely distributed sources in this case. The low-frequency array is able to detect a secondary lobe around the light dashdotted line, as can be seen in Fig. 5, which is principally produced by the trailing edge. The rough plates also generate stronger trailing-edge noise than the smooth plate, as predicted by Liu and Dowling [7], because on a rough plate the friction velocity $u_{\tau}$ and boundary-layer thickness $\delta$ are increased due to the enhanced surface drag and turbulence production [19].

However, we notice that the high-frequency array predicts 8-9 dB higher maximum SPL than the low-frequency array at all frequencies. A possible explanation is that the beamforming algorithm assumes a monopole source with uniform directivity, and that the locations of the array microphones are different. The microphones of the high-frequency array are confined in a relatively small region where considerable sound radiation can be received from the rough region upstream, while the microphones of the low-frequency array are distributed in a much wider area and thus some of them are located close to the $z$-axis where the roughness dipoles radiate little sound. The other and perhaps more important reason is based on the combination of the distributed 
nature of roughness sources and the difference in the resolution of both arrays. The high-frequency array has poorer resolution than the low-frequency array at the chosen frequencies, and hence tends to capture more roughness sources and add up their source levels.

\subsection{Effect of array locations}

Table 2

Locations of the array centre.

\begin{tabular}{cccc}
\hline Location no. & $x(\mathrm{~m})$ & $y(\mathrm{~m})$ & $z(\mathrm{~m})$ \\
\hline 1 & 0.04 & 0.025 & 0.47 \\
2 & 0.18 & 0.025 & 0.64 \\
3 & 0.36 & 0.025 & 0.60 \\
\hline
\end{tabular}

The sound radiation from the two rough plates, Rough1 and Rough2, was measured at three streamwise locations to detect some directivity features of the dipole sources. The coordinates of the array centre for locations 1-3 are listed in Table 2 with the origin $O$ at the centre of the rough region (see Fig. 8). In the $x$-direction, location 1 is very close to the origin $O$, location 2 is a bit downstream, and location 3 is further downstream and behind the rear edge of the rough region. Location 1 is also closest to the origin $O$ in the $z$-direction. In these measurements, four rough panels were used and so the rough region is doubled in area compared with that in Figs. 4 and 5.

Fig. 6 shows the beamforming source maps of Rough1 and Rough2 obtained by the high-frequency array at locations 1-3, respectively. As can be seen from Fig. 6, location 1 produces a major lobe upstream with a secondary lobe downstream. The minimum source strength lies in the middle of the rough region. This is very close to the centre of the array and occurs because the dipoles do not radiate sound in their normal plane. At the chosen frequency $f=2000 \mathrm{~Hz}$, the secondary lobe of Rough2 is stronger than that of Rough1 and covers a larger area, which has been predicted by the noise spectra comparison in Fig. 3. In contrast, only the distributed major lobe exists in the source maps at downstream locations 2 and 3. Higher maximum strengths can be observed as the array shifts downstream from location 1 to locations 2 and 3. All these features agree with the directivity characteristics of a distribution of dipole sources in the flow direction.

Comparing the distributed area of the major lobe at locations 1-3, we notice that the beamforming resolution becomes gradually worse as the array moves farther from the origin $O$. However at the nearest location, least radiated roughness noise is received by the microphone array due to the dipole 
(a)

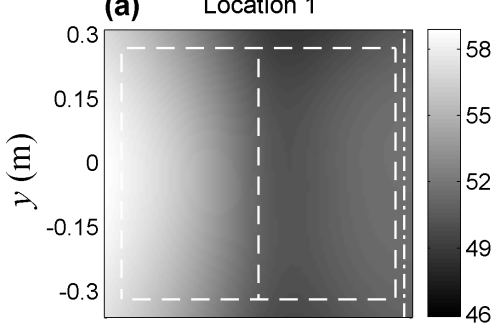

(b)

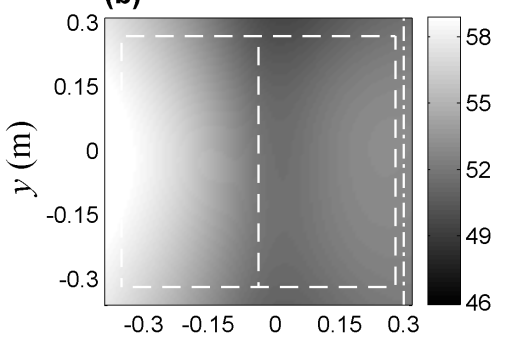

Location 2
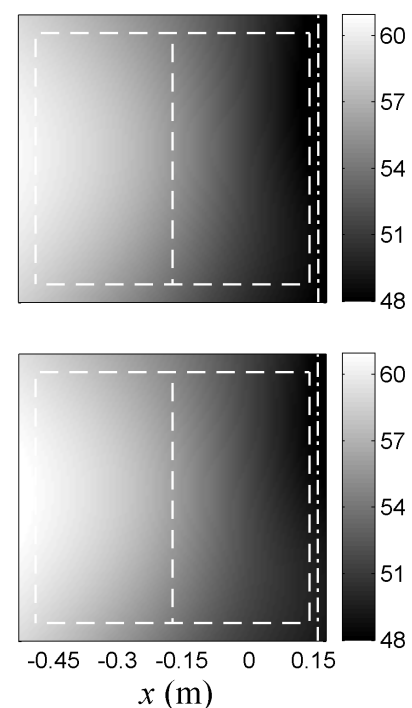

Location 3
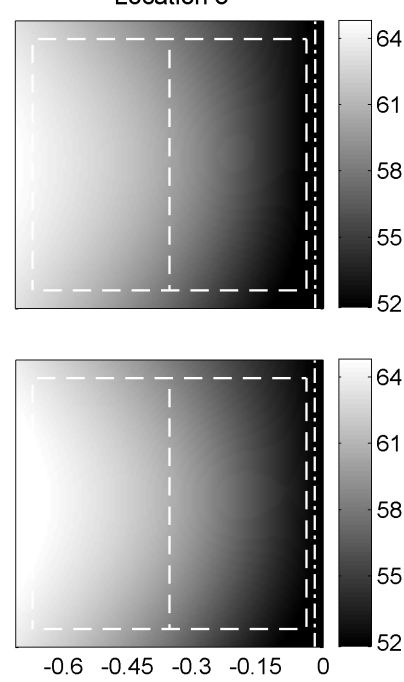

Fig. 6. Comparison of beamforming source maps at locations 1-3: (a) Rough1; (b) Rough2. High-frequency microphone array. $f=2000 \mathrm{~Hz}$.

directivity. Therefore, the compromise solution is to choose an array location a bit downstream from the central rough region, and this explains why location 2 was used for the measurements in $§ 3.2$.

\section{Theory}

Liu and Dowling [7] have given the power spectral density $P_{R}(\omega)$ of the far-field radiated roughness noise spectrum in the form:

$$
P_{R}(\omega)=\frac{A \sigma \mu^{2}}{4 r^{2}} \frac{R^{4}}{\delta^{* 4}} \frac{U_{c}^{2}}{c^{2}} \Phi(\omega) D(\theta, \phi)
$$

In the above expression, $A$ is the area of the rough region, the roughness density $\sigma=N \pi R^{2}$ means the fractional area of the plane covered by roughness elements, and $N$ is the average number of roughness elements per unit area. $\mu=1 /\left(1+\frac{1}{4} \sigma\right)$ is not appreciably different from unity. The convection velocity $U_{c} \approx 0.6 U$, and the displacement boundary-layer thickness $\delta^{*} \approx \delta / 8$ for practical purposes. $\Phi(\omega)$ is the point pressure frequency spectrum approximated by Ahn [20] for the frequency spectrum data in Blake [21]:

$$
\Phi(\omega)=\left(\frac{\tau_{w}^{2} \delta^{*}}{U}\right) \frac{2 \pi 8.28 S h^{* 0.8}}{\left[1+4.1 S h^{* 1.7}+4.4 \times 10^{-4} S h^{* 5.9}\right]},
$$




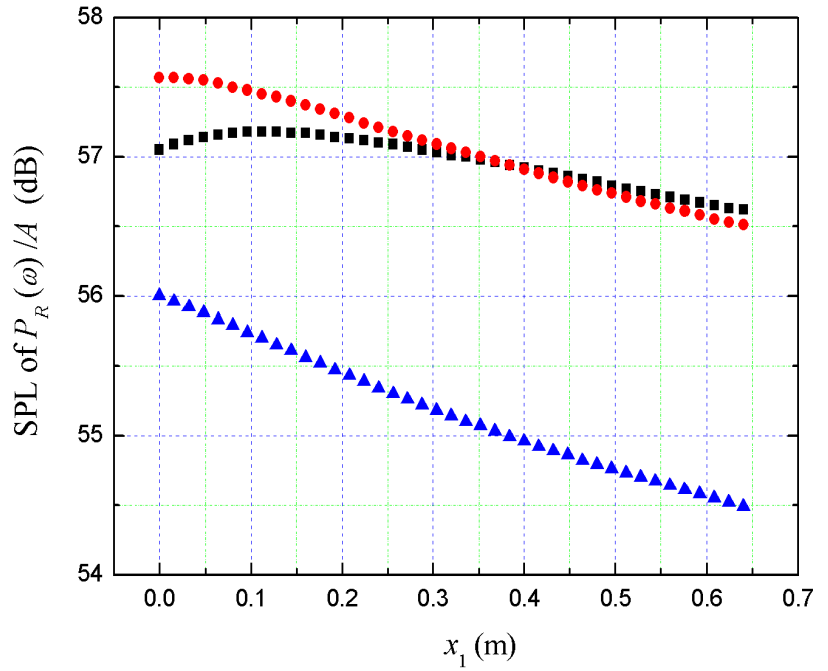

(a)

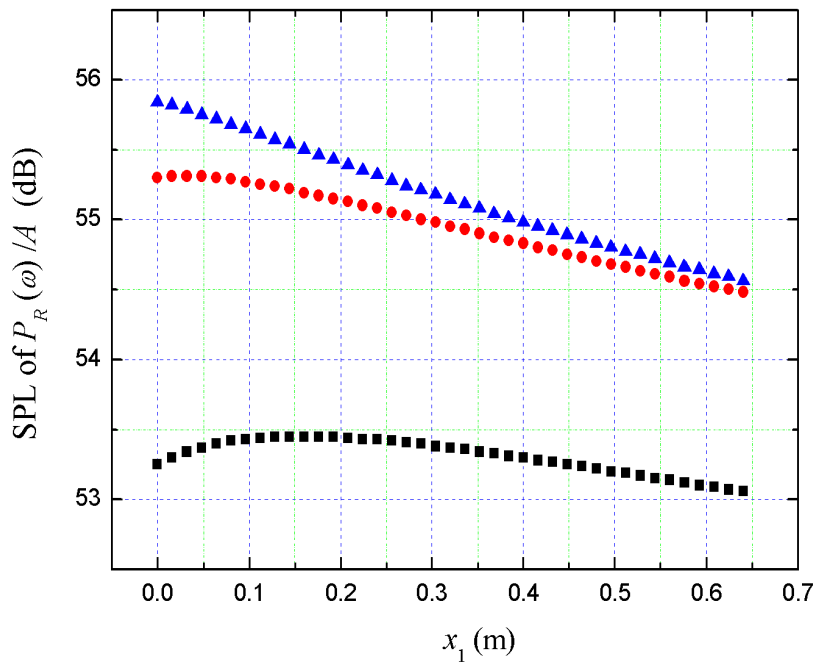

(b)

Fig. 7. Predicted SPL of $P_{R}(\omega) / A$ with streamwise distance $x_{1}$ for chosen frequencies: $1250 \mathrm{~Hz}, \bullet 1600 \mathrm{~Hz}, \boldsymbol{\Delta} 2000 \mathrm{~Hz}$. (a) Rough1; (b) Rough2.

where $\tau_{w}=\rho_{0} u_{\tau}^{2}$ is the mean wall shear stress, $u_{\tau}$ the friction velocity and $S h^{*}=\omega \delta^{*} / U$ the Strouhal number. $D(\theta, \phi)$ is the directivity function:

$$
D(\theta, \phi)=I_{1} \cos ^{2} \theta+I_{2} \sin ^{2} \theta \sin ^{2} \phi
$$

where $I_{1}$ and $I_{2}$ are infinite double integrals with respect to the wavenumber vector $\boldsymbol{\kappa}=\left(\kappa_{1}, 0, \kappa_{3}\right)$ (details to be found in Ref. [7]), and $\theta$ and $\phi$ are the directivity angles shown in Fig. 8 with

$$
0 \leqslant \theta \leqslant \pi \quad \text { and } \quad|\phi| \leqslant \pi / 2 \text {. }
$$

The prediction scheme in Eq. (1) is applied to calculate sound radiation from 
different streamwise portions of the two rough plates in the experiments. The theoretical results are for the power spectral density $P_{R}(\omega)$ from a unit rough area to an observer at

$$
\theta=\pi / 4, \quad \phi=0 \quad \text { and } \quad r=1 / \sqrt{4 \pi} \mathrm{m} .
$$

Figure 7 shows the predicted SPL of $P_{R}(\omega) / A$ distributed over the two rough plates at 1250, 1600 and $2000 \mathrm{~Hz}$ frequencies. At the two higher frequencies, $P_{R}(\omega) / A$ decreases with streamwise distance $x_{1}$ from the front edge of rough region. At $1250 \mathrm{~Hz}$, there is a maximum $x_{1}$ at $0.11 \mathrm{~m}$ for the plate Rough1 and at $0.16 \mathrm{~m}$ for Rough2.

On a rough plate, as the boundary layer grows along the chord ( $x$-axis), the local boundary-layer properties $\delta^{*}$ and $u_{\tau}$ are increasing and decreasing, respectively, both of which are determined by $x_{1}$. The overall dependence of $P_{R}(\omega) / A$ on $x_{1}$ at a particular frequency is principally due to the variation of $\Phi(\omega)$. More detailed investigation of the terms shows that the variation of $\Phi(\omega)$ accounts for the maximum of $P_{R}(\omega) / A$. At a frequency of $1250 \mathrm{~Hz}, \Phi(\omega)$ has a maximum at $x_{1}=0.11 \mathrm{~m}$ for Rough1 and at $x_{1}=0.16 \mathrm{~m}$ for Rough2. At the higher frequencies $\Phi(\omega)$ decreases across the entire rough regions.

\section{Comparison of theory and experiment}

\subsection{Motivation of theoretical simulation}

The comparison of beamforming source maps, as discussed in $\S 3.2$, demonstrates that the rough plates produce distinctly stronger noise sources than the smooth plate and enhance the trailing-edge noise somewhat. However, these "source" maps are not a true representation of the locations and relative strengths of the roughness dipoles because the beamforming algorithm assumes a distribution of monopole sources, and hence can not be used directly to validate the theoretical prediction.

Jordan et al. [16] has shown that the standard beamforming technique is inadequate for both the source location and the measurement of a simple dipole, and that this is due to the assumption of monopole propagation in the calculation of the phase weights used to steer the focus of the array. They developed a correction to the beamforming algorithm to account for the dipole propagation characteristics, and applied it to array measurements for an aeroacoustic dipole produced by a cylinder in a cross flow. The true source location and source energy of the dipole was then retrieved in the resulting source map after applying this correction. 
The technique of Jordan et al. [16], however, is not applicable in the case of a distribution of dipoles because their directivities and hence the required corrections vary over the source region, unlike the case of a single dipole. We do not have access to the source code for array analysis software to modify the algorithm for a distribution of axial dipoles. Furthermore, in practice the source mechanisms of a general aeroacoustic system could be very complex. There might be a combination of both monopole and dipole sources, and the dipoles may have axial and spanwise components. Therefore it would be difficult to implement a beamformer consistent with the hypothesized type of sources.

Instead of altering the beamformer, an indirect approach is to theoretically simulate a distribution of incoherent dipoles over a rigid plate using the prediction scheme of Liu and Dowling [7], to process the predicted sound field through the same algorithm as the experiment, and to generate predicted source maps that can be directly compared with the experimental results. This is equivalent to comparing theory and experiment after applying a filter which suppresses much the extraneous noise in the experiments. It provides an indirect way of comparing all the theoretical and experimental cross-powers between microphone pairs. For each grid point on the source-scanning plane, we determine the monopole source strength that gives the best fit to all the cross-powers. The quantitative agreement between the best-fit monopoles over the source plane for the theoretical and experimental cross-powers validates the prediction scheme.

\subsection{Simulation overview}

The theoretical simulation for an experiment using phased microphone arrays is illustrated in Fig. 8. A program SIMSRC was utilized to describe a distribution of incoherent dipoles over the rigid plate and simulate the sound detected by the microphone array, as exactly in the experimental setup. This program requires the original source locations and source strengths as input parameters. The post-processing and beamforming analysis of the simulation are based on a monopole source assumption as previously mentioned, and thus are not directly applicable to the dipole case of roughness noise. Nevertheless, SIMSRC is able to generate cross-power data for incoherent groups of coherent monopole sources [17]. In this case, each dipole source can be modeled by coherent pairs of closely spaced monopoles with opposite phase. Because the rigid plate behaves as a passive reflector, the mirror sources were also taken into account as coherent with the original sources. The distribution of incoherent dipoles was therefore modeled by incoherent groups of the four coherent monopole sets. 


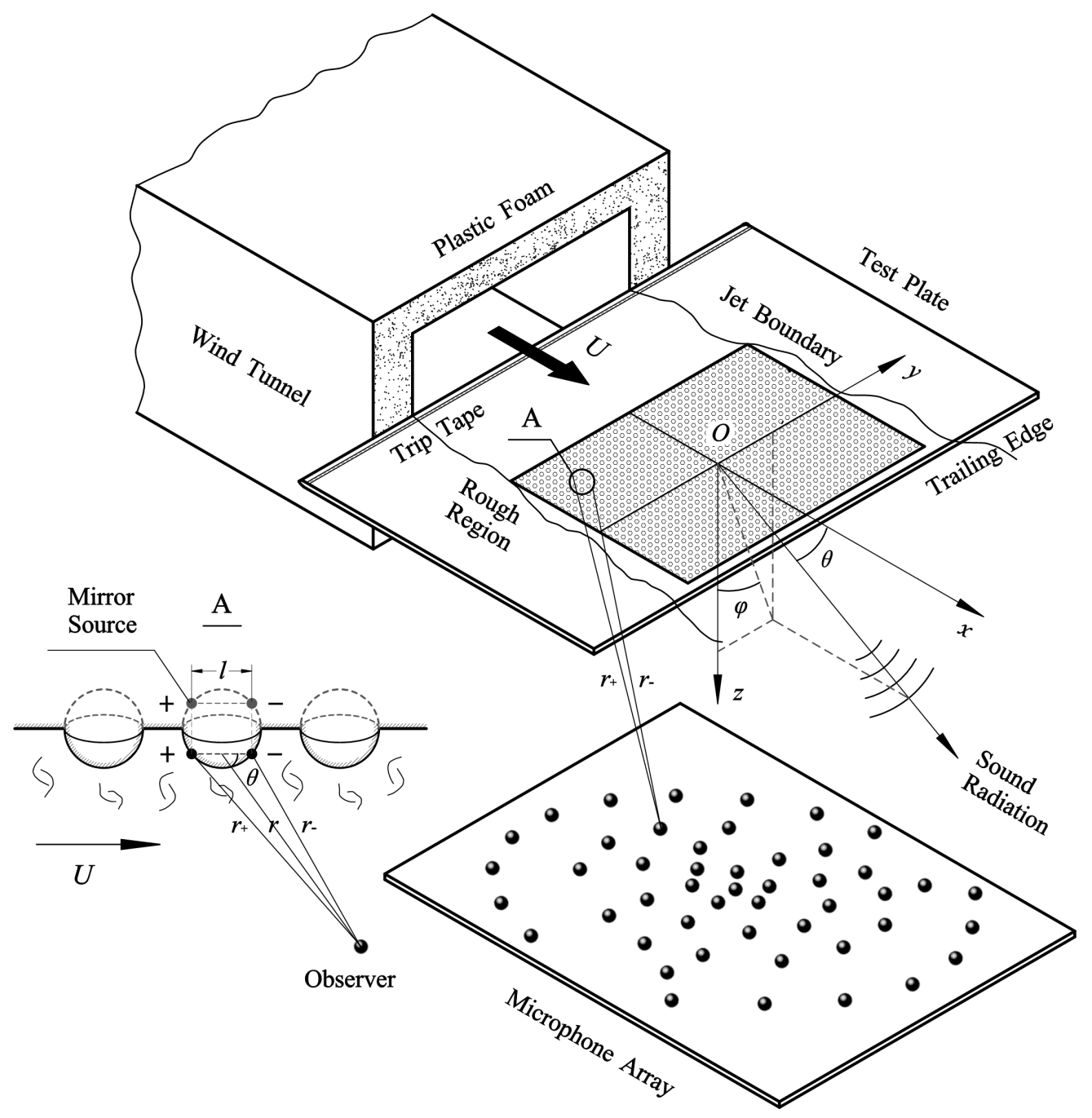

Fig. 8. Schematic of the theoretical simulation.

The phased microphone array is generally used to detect the source locations and source patterns, and the SPL data shown in source maps are usually obtained as relative and just for reference. In this study, however, we attempted to simulate the real source strengths in magnitude as well as the source locations. The simulated dipole sources were located at each hemispherical boss and the equivalent source strengths were determined from the prediction scheme of Liu and Dowling [7] as described below. The simulated acoustic field was then processed in the same way as in the experiments to obtain predicted beamforming source maps.

We now commence the determination of equivalent source strengths with the derivation of the acoustic field of a dipole source. The mean flow effects have been neglected since the prediction scheme is based on an assumption of low Mach number which is also satisfied in the experimental setup $(M<0.1)$. The shear-layer refraction has therefore not been considered in the derivation, neither. 


\subsection{Acoustic field of a dipole}

The acoustic frequency spectrum for an ideal monopole in a medium without flow can be expressed as [22]:

$$
\hat{p}(\omega)=\frac{-a(\omega)}{4 \pi r} \mathrm{e}^{-\mathrm{i} k r},
$$

where $a(\omega)$ is the monopole strength in frequency domain, $k=\omega / c$ is the acoustic wavenumber, and $r$ is the propagation distance from source to observer.

A dipole source can be modeled as a coherent pair of closely placed monopoles with opposite phase at a distance $l$ apart, as shown in Fig. 8. The acoustic field of a dipole is obtained by combining the radiated sound of these two monopoles:

$$
\hat{p}(\omega)=\frac{-a(\omega)}{4 \pi r_{+}} \mathrm{e}^{-\mathrm{i} k r_{+}}-\frac{-a(\omega)}{4 \pi r_{-}} \mathrm{e}^{-\mathrm{i} k r_{-}},
$$

where $r_{+}$and $r_{-}$are the propagation distances for the two monopoles with opposite phase,

$$
\left\{\begin{array}{l}
r_{+} \approx r+\frac{l}{2} \cos \theta, \\
r_{-} \approx r-\frac{l}{2} \cos \theta .
\end{array}\right.
$$

In the far field, $r \gg l$, the amplitude difference between the radiated sound of two monopoles is small, and thus in Eq. (7) $r_{+}$and $r_{-}$can be approximated by $r$ in the amplitude part. However, the phase difference can not be ignored. Substituting Eq. (8) into Eq. (7), we obtain

$$
\begin{aligned}
\hat{p}(\omega) & \approx \frac{-a(\omega)}{4 \pi r} \mathrm{e}^{-\mathrm{i} k r} \cdot\left(\mathrm{e}^{-\mathrm{i} \frac{k l \cos \theta}{2}}-\mathrm{e}^{\mathrm{i} \frac{k l \cos \theta}{2}}\right) \\
& =\frac{-a(\omega)}{4 \pi r} \mathrm{e}^{-\mathrm{i} k r} \cdot\left(-2 \mathrm{i} \sin \frac{k l \cos \theta}{2}\right) .
\end{aligned}
$$

If the dipole is compact (i.e. $k l \ll 1$ ), Eq. (9) can be simplified as:

$$
\hat{p}(\omega)=\frac{a(\omega)}{4 \pi r} \mathrm{e}^{-\mathrm{i} k r} \cdot \mathrm{i} k l \cos \theta
$$

In the presence of a reflecting rigid plate, the mirror source of the dipole need to be included, and hence the aggregate acoustic field can be obtained by multiplying Eq. (10) by 2. The power spectral density of the acoustic frequency spectrum $\hat{p}(\omega)$ is:

$$
P(\omega)=\Lambda(\omega)\left|\frac{\mathrm{i} k l \cos \theta}{2 \pi r} \mathrm{e}^{-\mathrm{i} k r}\right|^{2}
$$


where $\Lambda(\omega)$ is the power spectral density of $a(\omega)$,

$$
\overline{a(\omega) a\left(\omega^{\prime}\right)}=2 \pi \Lambda(\omega) \delta\left(\omega+\omega^{\prime}\right) .
$$

\subsection{Equivalent source strengths}

As is evident from Eq. (3), the first term $I_{1} \cos ^{2} \theta$ describes the sound field due to a dipole in the flow direction, while the second term $I_{2} \sin ^{2} \theta \sin ^{2} \phi$ accounts for a dipole in the plate plane but normal to the flow direction. To link this to the beamforming simulation, we consider a distribution of dipoles with two dipoles $\mathrm{DPL}_{1}$ and $\mathrm{DPL}_{2}$ at each hemispherical boss and determine the equivalent source strengths $a_{1}(\omega)$ and $a_{2}(\omega)$. Herein DPL $\mathrm{D}_{1}$ is orientated in the flow direction and $\mathrm{DPL}_{2}$ is normal to the flow direction, respectively.

Now we consider a rough region of unit area which contains $N$ roughness elements. The acoustic field of $\mathrm{DPL}_{1}$ can be described by Eq. (11), and hence the aggregate power spectral density of $N$ incoherent dipoles is

$$
P_{1}(\omega)=\sum_{j=1}^{N} P_{1 j}(\omega)=\frac{N \Lambda_{1}(\omega) k^{2} l^{2} \cos ^{2} \theta}{4 \pi^{2} r^{2}} .
$$

From the prediction scheme (1), the contribution of the dipole $\mathrm{DPL}_{1}$ to $P_{R}(\omega)$ for a unit rough area $A=1$ is

$$
P_{R 1}(\omega)=\frac{\sigma \mu^{2}}{4 r^{2}} \frac{R^{4}}{\delta^{*}} \frac{U_{c}^{2}}{c^{2}} \Phi(\omega) I_{1} \cos ^{2} \theta
$$

Combining Eqs. (13) and (14) gives the theoretical prediction for $\Lambda_{1}(\omega)$ :

$$
\Lambda_{1}(\omega)=\frac{\pi^{2} \sigma \mu^{2}}{N l^{2}} \frac{R^{4}}{\delta^{*}} \frac{U_{c}^{2}}{\omega^{2}} \Phi(\omega) I_{1} .
$$

The above derivation is based on the continuous Fourier transform (CFT) and $\Lambda_{1}(\omega)$ denotes the power spectral density of $a_{1}(\omega)$. However in the theoretical simulation for acoustic measurements, the discrete Fourier transform (DFT) is applied and thus $\Lambda_{1}(\omega)$ actually means the frequency-dependent source power [22]. In this case, the equivalent source strength $a_{1}(\omega)$ required by the simulation program SIMSRC can not be derived directly from $\Lambda_{1}(\omega)$ in Eq. (15). Instead, it is necessary to compare the total source power in a frequency band between the CFT and DFT.

The predicted total power of $\Lambda_{1}(\omega)$ in the frequency band $\left[\omega_{1}, \omega_{n}\right]$ is

$$
\overline{a_{1}^{2}(t)}=\frac{1}{2 \pi} \int_{\omega_{1}}^{\omega_{n}} \Lambda_{1}(\omega) \mathrm{d} \omega .
$$


In the simulation, the total power of $\Lambda_{1}(\omega)$ in $\left[\omega_{1}, \omega_{n}\right]$ can be expressed as [23]:

$$
\Lambda_{1 \mathrm{tot}}=\sum_{i=1}^{n}\left|a_{1}\left(\omega_{i}\right)\right|^{2}=n\left|\bar{a}_{1}(\omega)\right|^{2}
$$

where $n$ is the number of frequency intervals; $a_{1}\left(\omega_{i}\right)$ is the equivalent source strength in the $i$ th frequency interval; $\bar{a}_{1}(\omega)$ is the average source strength of $n$ intervals, $\omega_{1}, \omega_{2}, \ldots, \omega_{n}$, and is used as the input source strength for the frequency band $\left[\omega_{1}, \omega_{n}\right]$ in SIMSRC. By equating the total source powers in the prediction (16) and simulation (17), the predicted equivalent source strength for the two coherent monopoles of $\mathrm{DPL}_{1}$ is given by

$$
\left|\bar{a}_{1}(\omega)\right|=\frac{\mu U_{c}}{l} \frac{R^{2}}{\delta^{* 2}}\left[\frac{\pi \sigma I_{1}}{2 N n} \int_{\omega_{1}}^{\omega_{n}} \frac{\Phi(\omega)}{\omega^{2}} \mathrm{~d} \omega\right]^{1 / 2} .
$$

Just as the power spectral density $P_{R}(\omega)$, the equivalent source strength $\bar{a}_{1}(\omega)$ decreases with increasing streamwise position along the rough region, except at the lowest frequency $f=1250 \mathrm{~Hz}$ where it has a maximum near $x_{1}=0.11 \mathrm{~m}$ for the plate Rough1 and $0.16 \mathrm{~m}$ for Rough2. Equation (18) describes the variation of the roughness dipole strength with streamwise locations. The value of the dipole size $l$ is unimportant because $\Lambda_{1}(\omega) \sim l^{-2}$ from Eq. (15) and hence the predicted power spectral density $P_{1}(\omega)$ is independent of $l$. The only constraint is that $l$ should satisfy the compact dipole assumption $k l \ll 1$. In the present study $l=R$ is used.

Similarly, the theoretical prediction for $\left|\bar{a}_{2}(\omega)\right|$ can be obtained as:

$$
\left|\bar{a}_{2}(\omega)\right|=\frac{\mu U_{c}}{l} \frac{R^{2}}{\delta^{*^{2}}}\left[\frac{\pi \sigma I_{2}}{2 N n} \int_{\omega_{1}}^{\omega_{n}} \frac{\Phi(\omega)}{\omega^{2}} \mathrm{~d} \omega\right]^{1 / 2} .
$$

Nevertheless, the contribution of $I_{1}$ to $P_{R}(\omega)$ is more important than that of $I_{2}$ for sufficiently large roughness elements [7], i.e. the roughness Reynolds number

$$
R e_{\tau}=R u_{\tau} / \nu>1000
$$

where $\nu$ is the kinematic viscosity. In addition, in Fig. 8 the centre of the microphone array is located very close to the centre of the rough region in the $y$-direction which is the direction of the dipole $\mathrm{DPL}_{2}$. This results in a nearly negligible contribution of $\mathrm{DPL}_{2}$ to the SPL of the source maps as there is no sound radiation in the normal plane of the $\mathrm{DPL}_{2}$ orientation. The predicted $\left|\bar{a}_{1}(\omega)\right|$ and $\left|\bar{a}_{2}(\omega)\right|$ from Eqs. (18) and (19) were then used in the theoretical simulation as the equivalent source strengths for $\mathrm{DPL}_{1}$ and $\mathrm{DPL}_{2}$, respectively. 


\subsection{Results}

To compare the source maps of measured roughness noise and simulated dipole sources, "clean" source maps of Rough1 and Rough2 need to be obtained. Although the reverberation noise of a closed-return wind tunnel is avoided in the case of out-of-flow measurements in an open jet, Figs. 4 and 5 still indicate considerable contamination from other sound sources, e.g. trailing edge, leading edge, seams. A straightforward but effective method to eliminate the contamination is to subtract the source powers of the smooth plate from those of the rough plates. This method was applied to the raw source maps in Figs. 4 and 5, and corrected "clean" source maps were obtained for comparison with simulation.

Figs. 9-12 illustrate the comparison of measured and simulated source maps for Rough1 and Rough2, and both the high- and low-frequency array data are shown. Unlike the identical grey-scale bars for Rough1, Rough2 and Smooth in Figs. 4 and 5, the grey-scale bars in Figs. 9-12 gives the unaltered maximum source strengths for a better comparison of measurement and simulation. The simulated equivalent source strengths at each $1 / 3$ octave band frequency have been averaged over the whole bandwidth, as in the beamforming analysis of the experimental data.

In all these figures, the top row shows the "clean" source maps based on the experimental data with the Smooth source powers subtracted. As can be seen from the high-frequency array data in Figs. 9 and 11, the SPL of the "clean" source maps has been diminished a bit and the major lobe is concentrated more in the upstream rough region compared with the raw source maps in Fig. 4. This correction is shown more evidently for the low-frequency array data in Figs. 10 and 12 in which the interference from the trailing-edge noise seen originally in Fig. 5 has been greatly reduced.

The bottom row of Figs. 9-12 shows the corresponding source maps by simulating a distribution of incoherent dipoles over the rigid plate with strengths derived using the model of Liu and Dowling [7]. The beamforming predictions of the source patterns of measurement and simulation exhibit satisfactory similarities, which confirms the dipole nature of surface roughness noise. The major lobe in the top row gradually reduces in the spanwise direction ( $y$-axis) along the plate chord ( $x$-axis), whereas in the bottom row the major lobe almost fills the entire dashed frame. This is because in the experiment the boundary of the open jet expands along the flow direction as the jet mixes with the still air in free space. The expansion effect results in a decrease of flow velocity around the jet boundary. In the simulation, however, this effect is too complicated to be considered for the correction of the predicted equivalent source strengths around the jet boundary. 

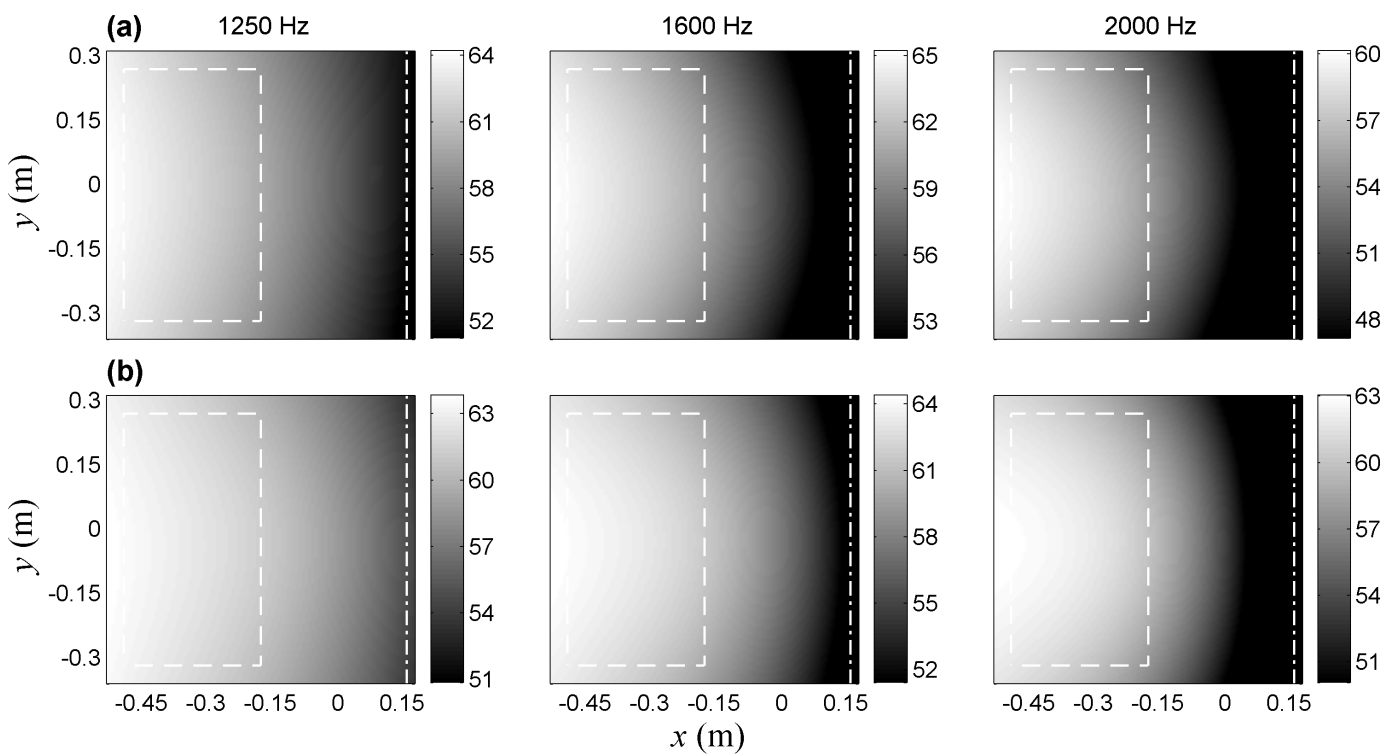

Fig. 9. Comparison of beamforming source maps for Rough1: (a) measurement ("clean"); (b) simulation. High-frequency microphone array.

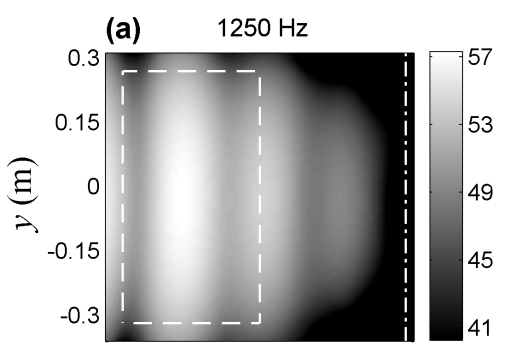

(b)

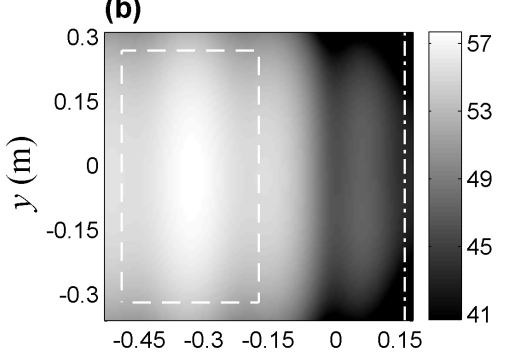

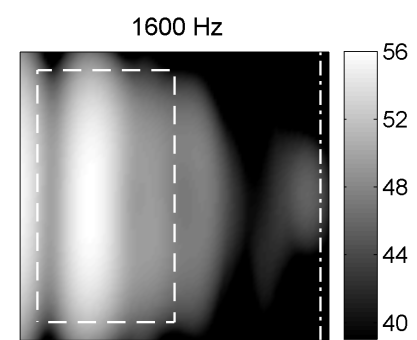
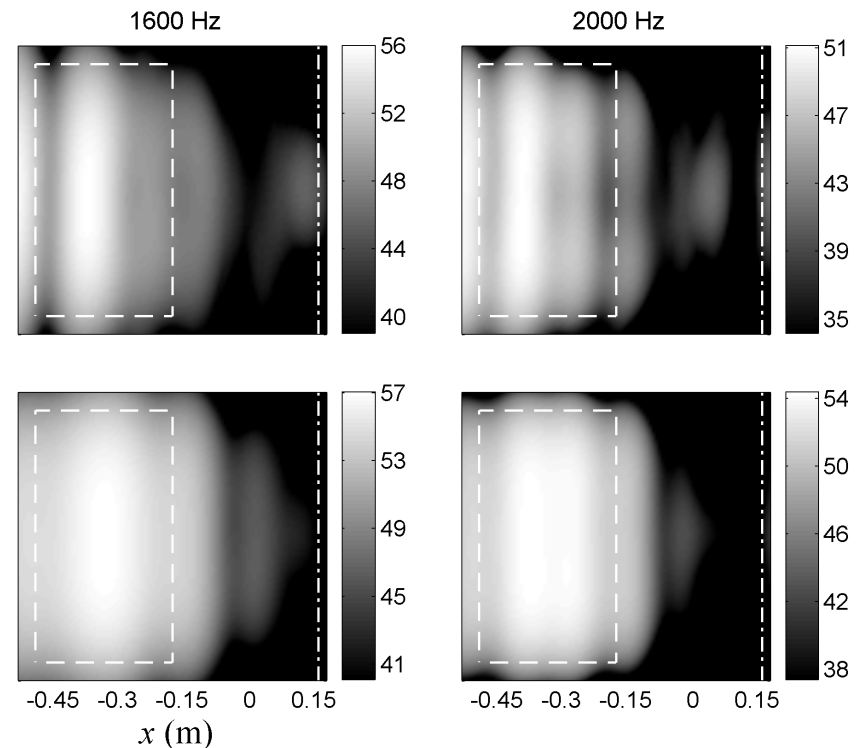

Fig. 10. Comparison of beamforming source maps for Rough1: (a) measurement ("clean"); (b) simulation. Low-frequency microphone array.

Furthermore, as indicated by the grey-scale bars of Figs. 9-12, the simulation program SIMSRC is capable of approximately predicting the equivalent source strengths of roughness noise in magnitude at 1250 and $1600 \mathrm{~Hz}$ frequencies, which provides further form of validation for Liu and Dowling's prediction scheme [7] from the perspective of microphone array measurements. However, a discrepancy of about $3 \mathrm{~dB}$ can be observed for the comparison at $2000 \mathrm{~Hz}$, and this should be ascribed to the limitation of Liu and Dowling's prediction scheme which predicted spectral levels a few $\mathrm{dB}$ higher than the measured 

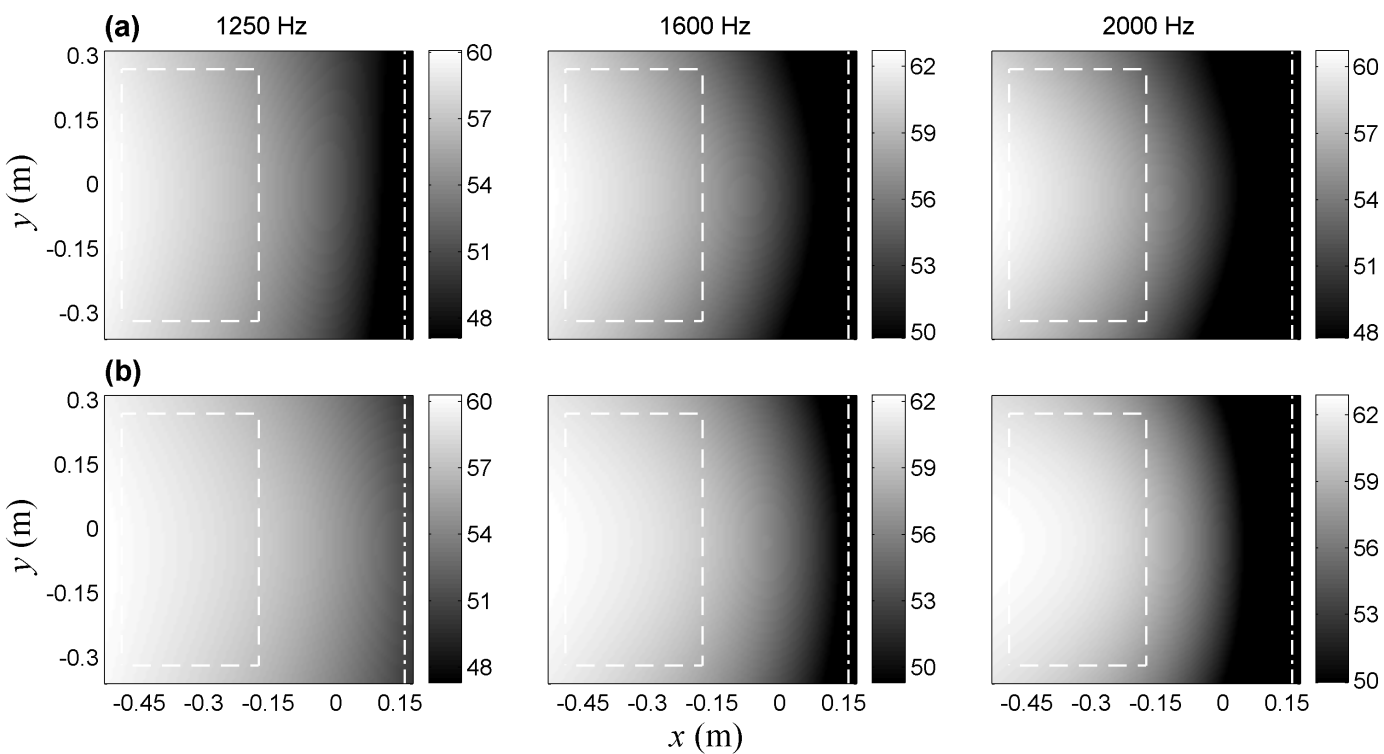

Fig. 11. Comparison of beamforming source maps for Rough2: (a) measurement ("clean"); (b) simulation. High-frequency microphone array.
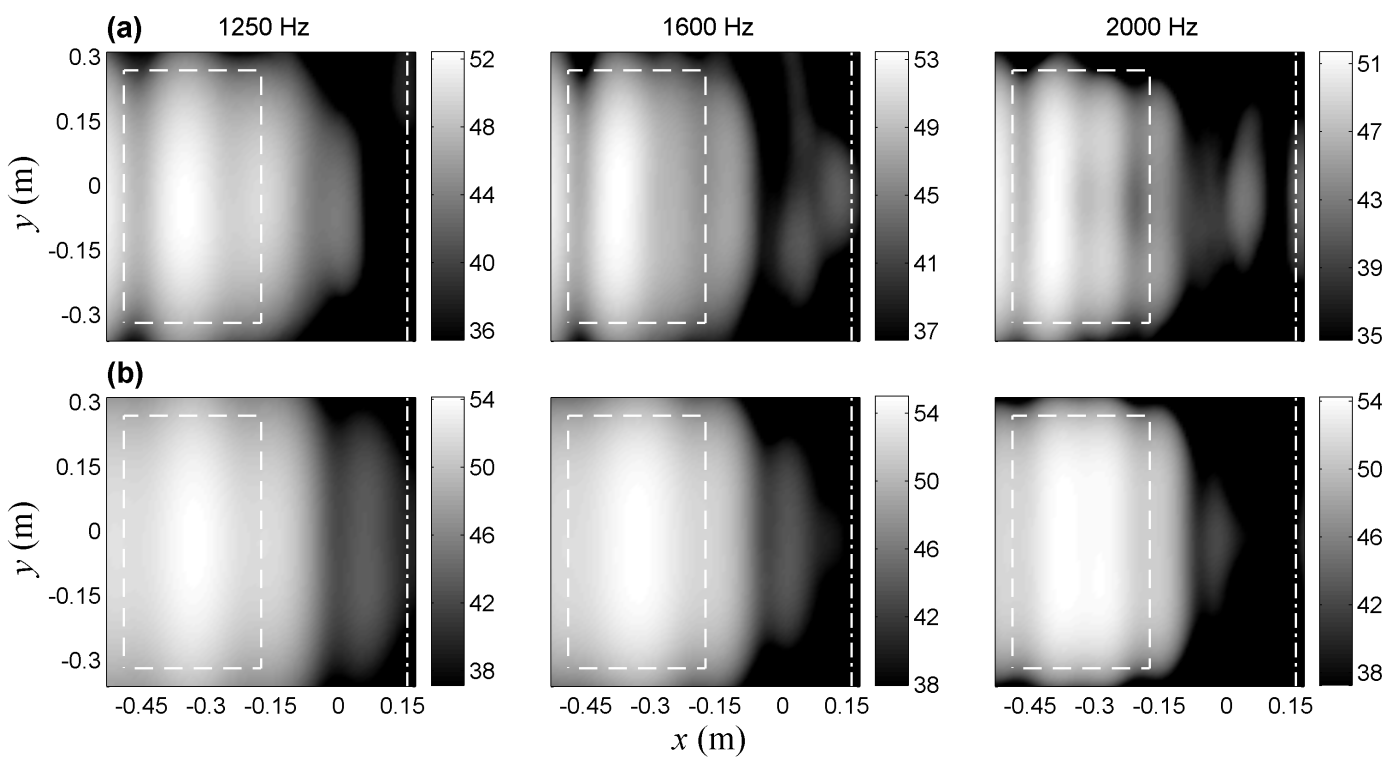

Fig. 12. Comparison of beamforming source maps for Rough2: (a) measurement ("clean"); (b) simulation. Low-frequency microphone array.

roughness noise in $f>1.7 \mathrm{kHz}$ frequency [7]. In addition, SIMSRC predicts somewhat higher source strengths in the downstream portion of the rough region, namely, the streamwise gradient of simulated source strengths are a bit lower than that of the measured source strengths. Hence there is scope to improve the theoretical model to capture these aspects of surface roughness generated noise. 


\section{Concluding remarks}

Howe [1] has presented a theoretical model of sound generation by turbulent boundary-layer flow over a rough wall. The dipole-type roughness noise was attributed to the scattering of the turbulence near-field into radiated sound at small surface irregularities. Liu and Dowling [7] then extended Howe's model for numerically quantifying the far-field radiated roughness noise, and have obtained reasonable agreement between measurement and prediction in roughness noise spectral levels.

In this paper, phased microphone arrays have been applied to the measurement and simulation of surface roughness noise. From the resulting beamforming source maps, the rough plates exhibited higher source strengths than the smooth plate, and the trailing-edge noise was somewhat enhanced by surface roughness. Measurements at three streamwise locations demonstrated some features of the dipole directivity.

Theoretical simulations have been performed for a distribution of incoherent dipoles over the rough plates with the equivalent source strengths determined by Liu and Dowling's prediction scheme [7]. The same beamfroming algorithm was applied to measurement and simulation and the source maps exhibited satisfactory similarities in source pattern with approximate source strengths. This has confirmed the dipole nature of roughness noise and validated the source amplitude predicted by Liu and Dowling [7]. However, the streamwise gradient of the source strengths was a bit underestimated in the simulations, and at the highest frequency the source strengths were overestimated by about $3 \mathrm{~dB}$, which indicates that there is scope for an improved theoretical prediction which captures these aspects of surface roughness noise.

As well as a contribution to surface roughness noise, the proposed processing technique has wider applicability. For many airframe applications, the aeroacoustic sources arise from dipoles and quadrupoles. In this case, array measurements can be misinterpreted due to the monopole assumption in the standard beamforming algorithm. We analyzed the theoretical model through the array simulation software so that indirect comparison between theory and experiment can be made for the dipole-type surface roughness noise. It is recommended that further investigations be conducted to apply this technique to more complex systems, for example, quadrupole-type jet noise. 


\section{Acknowledgements}

This work is supported in part by Silent Aircraft Initiative (SAI), a collaborative project funded by the Cambridge-MIT Institute. The design and manufacture of the microphone array system was assisted by National Aerospace Laboratory (NLR), The Netherlands. The authors would like to thank Dr. Pieter Sijtsma of NLR for the technical support to the results obtained from the microphone arrays. Y. Liu acknowledges the financial support provided by the Overseas Research Students (ORS) Awards Scheme and the Gates Cambridge Scholarships.

\section{References}

[1] M. S. Howe, On the generation of sound by turbulent boundary layer flow over a rough wall, Proceedings of the Royal Society of London A395 (1984) 247-263.

[2] M. S. Howe, The influence of viscous surface stress on the production of sound by a turbulent boundary layer over a rough wall, Journal of Sound and Vibration 104 (1) (1986) 29-39.

[3] M. S. Howe, The turbulent boundary-layer rough-wall pressure spectrum at acoustic and subconvective wavenumbers, Proceedings of the Royal Society of London A415 (1988) 141-161.

[4] M. S. Howe, Surface pressures and sound by turbulent flow over smooth and rough walls, Journal of the Acoustical Society of America 90 (2) (1991) 10411047.

[5] M. S. Howe, Acoustics of Fluid-Structure Interactions, Cambridge University Press, Cambridge, UK, 1998.

[6] A. S. Hersh, Surface roughness generated flow noise, No. 83-0786, AIAA Paper, 1983.

[7] Y. Liu, A. P. Dowling, Assessment of the contribution of surface roughness to airframe noise, AIAA Journal 45 (4) (2007) 855-869.

[8] G. M. Corcos, The structure of the turbulent pressure field in boundary- layer flows, Journal of Fluid Mechanics 18 (3) (1964) 353-378.

[9] B. M. Efimtsov, Characteristics of the field of turbulent wall pressure fluctuations at large reynolds numbers, Soviet Physics Acoustics 28 (4) (1982) 289-292.

[10] A. V. Smol'yakov, V. M. Tkachenko, Model of a field of pseudosonic turbulent wall pressures and experimental data, Soviet Physics Acoustics 37 (6) (1991) $627-631$. 
[11] D. M. Chase, Modeling the wavevector-frequency spectrum of turbulent boundary layer wall pressure, Journal of Sound and Vibration 70 (1) (1980) $29-67$.

[12] D. M. Chase, The character of the turbulent wall pressure spectrum at subconvective wavenumbers and a suggested comprehensive model, Journal of Sound and Vibration 112 (1) (1987) 125-147.

[13] J. Billingsley, R. Kinns, The acoustic telescope, Journal of Sound and Vibration 48 (4) (1976) 485-510.

[14] D. H. Johnson, D. E. Dudgeon, Array Signal Processing: Concepts and Techniques, Prentice Hall, London, 1993.

[15] R. P. Dougherty, Beamforming in Acoustic Testing, Published in "Aeroacoustic Measurements", Edited by T. J. Mueller, Springer-Verlag, Berlin, 2002.

[16] P. Jordan, J. A. Fitzpatrick, J.-C. Valière, Measurement of an aeroacoustic dipole using a linear microphone array, Journal of the Acoustical Society of America 111 (3) (2002) 1267-1273.

[17] P. Sijtsma, Experimental Techniques for Identification and Characterisation of Noise Sources, Published in "Advances in Aeroacoustics and Applications", VKI Lecture Series 2004-05, Edited by J. Anthoine and A. Hirschberg, 2004.

[18] R. K. Amiet, Refraction of sound by a shear layer, Journal of Sound and Vibration 58 (2) (1978) 467-482.

[19] H. Schlichting, Boundary Layer Theory, seventh Edition, McGraw-Hill, New York, 1979.

[20] B. Ahn, Modeling unsteady wall pressures beneath turbulent boundary layers, Ph.D. thesis, Department of Engineering, University of Cambridge, Cambridge, UK (2005).

[21] W. K. Blake, Turbulent bounday-layer wall-pressure fluctuations on smooth and rough walls, Journal of Fluid Mechanics 44 (4) (1970) 637-660.

[22] A. P. Dowling, J. E. Ffowcs Williams, Sound and Sources of Sound, Ellis Horwood, Chichester, UK, 1983.

[23] N. C. Geckinli, D. Yavuz, Discrete Fourier Transformation and Its Applications to Power Spectra Estimation, Published in "Studies in Electrical and Electronic Engineering 8", Elsevier, New York, 1983. 